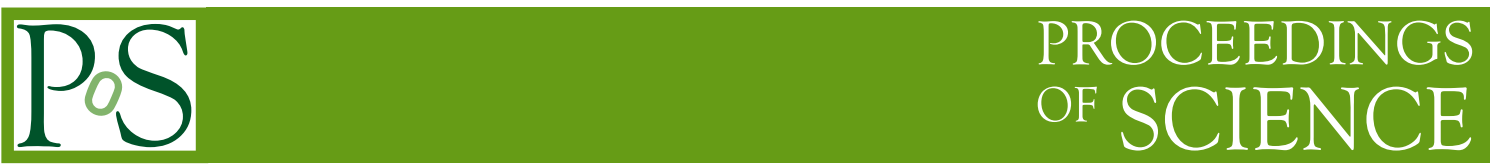

\title{
Gamma-ray emission from Seyfert galaxy NGC 1068
}

\author{
Giovanna Pedaletti \\ Institut de Ciencies de L'Espai (IEEC-CSIC) \\ E-mail: gpedaletdieec.uab.es
}

\section{Stefan J. Wagner}

\section{Landessternwarte Heidelberg}

NGC 1068 is a prototypical Seyfert galaxy, with an unusual optical spectrum detected already by [Fath(1909)]. Subsequent observation led to inclusion in the original list by [Seyfert(1943)]. With similar widths of permitted and forbidden lines, NGC 1068 was classified as a type 2 Seyfert galaxy, despite showing unusually broad lines in all transitions. Spectropolarimetry revealed permitted lines of much larger line width in polarized light. This suggested the presence of a bona-fide broad line region (BLR) hidden from direct observations by an opaque circumnuclear torus, but detectable through scattering in the optical thin regimes around the material causing the obscuration of the direct line-of-sight. Radio observations revealed two jets on sub-parsec through kiloparsec scales, but VLBI observations only found subrelativistic motion. The northern jet displays a prominent nose-cone, generally interpreted to be formed by a bow-shock driven by the jetted outflow into the interstellar medium of the host galaxy. The morphology of the polarization maps suggests compression of magnetic fields at this bow-shocks. The jet kinetic power of the system can be as high as $P_{\text {jet }} \sim 3 \times 10^{43} \mathrm{erg} \mathrm{s}^{-1}$ [Wilson \& Ulvestad(1987)].

25th Texas Symposium on Relativistic Astrophysics

December 6-10, 2010

Heidelberg, Germany 


\section{Introduction}

During the last two decades satellite-borne and ground-based gamma-ray instruments have detected high-energy ( $\mathrm{HE}, 100 \mathrm{MeV}<\mathrm{E}<100 \mathrm{GeV}$ ) and very high energy (VHE, E $>100 \mathrm{GeV}$ ) gamma-ray emission from AGN classified as Blazars. One common property of this class of sources is significant relativistic amplification of the non-thermal radiation due to bulk relativistic motion. The gamma-ray emission is likely to originate in the relativistic jets of these objects. High compactness, implied by short variability time-scales, can only be reconciled with the implication of low internal opacity for gamma-rays by relativistic amplification. In a number of sources, flares of gamma-ray emission have been associated with newly emerging knots within the jets, which, in turn, move with relativistic velocities. Apart from bona-fide Blazars, which are thought to be singled out by orientation, with the line-of-sight forming small angles to the axes of the relativistic jets of these AGN, a few systems were found to be gamma-ray bright which are believed to host relativistic jets pointing sideways (M87, Cen A). Even in such systems, however, relativistic motion is implied with VLBI observations. Despite orientation-implied biases there are many AGN which are powerful radio-emitters (directly indicating the presence of relativistic particles) which do not exhibit any bulk relativistic motion. It is important to understand whether powerful relativistic amplification is indeed required to explain the gamma-ray luminosity of nearby AGN. Gamma-ray emission may also result from particles accelerated in shocks, even if the bulk flow is non-relativistic. Bow-shocks generated in the interaction of jets with large kinetic energies pushing through dense ISM have been studied observationally, numerically, and theoretically. Here we discuss gamma-ray observations of NGC 1068, a prototypical Seyfert 2 galaxy. An association of a Fermi excess with NGC 1068 has already been explored by [Lenain et al.(2010)].

\section{Fermi Analysis Details}

The LAT instrument onboard the Fermi Gamma-ray Space Telescope provides an all-sky scan in the high energy range $\left(100 \mathrm{MeV}<\mathrm{E}_{\mathrm{Fermi}}<300 \mathrm{GeV}\right)$. A catalog of Fermi sources, based on the first eleven months of data, has been released and comprises 1451 sources above a significance threshold of about $4 \sigma$ (standard deviations). Among these, the source 1FGL J0242.7+0007, 5.2 $\sigma$ at the position $(\mathrm{RA}, \mathrm{DEC})=(2 \mathrm{~h} 42 \mathrm{~m} 42.6 \mathrm{~s},+0 \mathrm{~d} 07 \mathrm{~m} 58.1 \mathrm{~s})$ is spatially coincident with the position of the active nucleus of NGC $1068(2 \mathrm{~h} 42 \mathrm{~m} 40.8 \mathrm{~s},-0 \mathrm{~d} 00 \mathrm{~m} 48.4 \mathrm{~s})$. The offset is of 8.8'. In this work, public data of the LAT instrument taken during the period 04 August 2008 - 15 November 2010 are analyzed in detail using the public software released by the Fermi Collaboration (v9r15p2fssc090808). Only events between $200 \mathrm{MeV}$ and $100 \mathrm{GeV}$ were considered and standard selection cuts for point-sources were applied. As outlined in the Cicerone Fermi manual, the region whose counts are considered in the analysis has been chosen to be of $15^{\circ}$ (RoI, Region of Interest). This size of the region is standard for sources whose flux is dominated at around $1 \mathrm{GeV}$. In the modeling of the counts, all sources from the 1FGL source catalog in a bigger region of $25^{\circ}$ (SR, Source Region) have been considered as being able to contribute to the counts in the RoI, due to the large tail of the instrument PSF at low energy. The source is detected with a test statistic TS $\sim 68$, corresponding to a significance of $\mathrm{S} \sim \sqrt{\mathrm{TS}} \sim 8 \sigma$ in the entire energy range, from an excess of 220 photons. In the SR there are 36 sources, of which 22 are in the RoI. 


\subsection{Localization Details}

The best fit position for the entire data set is $($ RA,DEC $)=(2 \mathrm{~h} 42 \mathrm{~m} 42.9 \mathrm{~s},+0 \mathrm{~d} 05 \mathrm{~m} 13.2 \mathrm{~s})$. The error circle radius is $5.4^{\prime}\left(68 \%\left(8.8^{\prime} 95 \%\right)\right.$, statistical only, the systematic error is $\left.2.4^{\prime}\right)$. The position has been found using the GTFINDSRC tool in the analysis package. In the full data set presented here, the gamma-ray source is positionally coincident with the position of the active nucleus of NGC 1068, with an offset of 5.6'. Our best fit position is inside the 11 months error ellipse from the Fermi catalog, with an offset of 3'. In order to check the reliability of the offset, we repeated the procedure on the same data set used for the 11 month catalog. We find the position ( $2 \mathrm{~h} 42 \mathrm{~m} 45.1 \mathrm{~s},+0 \mathrm{~d} 08 \mathrm{~m} 05.0 \mathrm{~s})$, virtually identical to the position of the catalog (0.6' off), as can be seen from Fig. $\square$. The best fit position and localization error can be refined, using only the data with $\mathrm{E}_{\text {phot }}>10 \mathrm{GeV}$, where photons can be localized with the highest precision. With this constraint, the best fit position is $(2 \mathrm{~h} 42 \mathrm{~m} 45 \mathrm{~s},-0 \mathrm{~d} 00 \mathrm{~m} 36 \mathrm{~s})$ with an error circle radius of 5.3' $\left(68 \%\left(8.7^{\prime} 95 \%\right)\right.$ statistical only). The offset of the nominal position of NGC1068 in this case is of 1.5', that is a small fraction of the localization circle. Best fit localizations and errors are shown in Fig. $\mathrm{W}$.

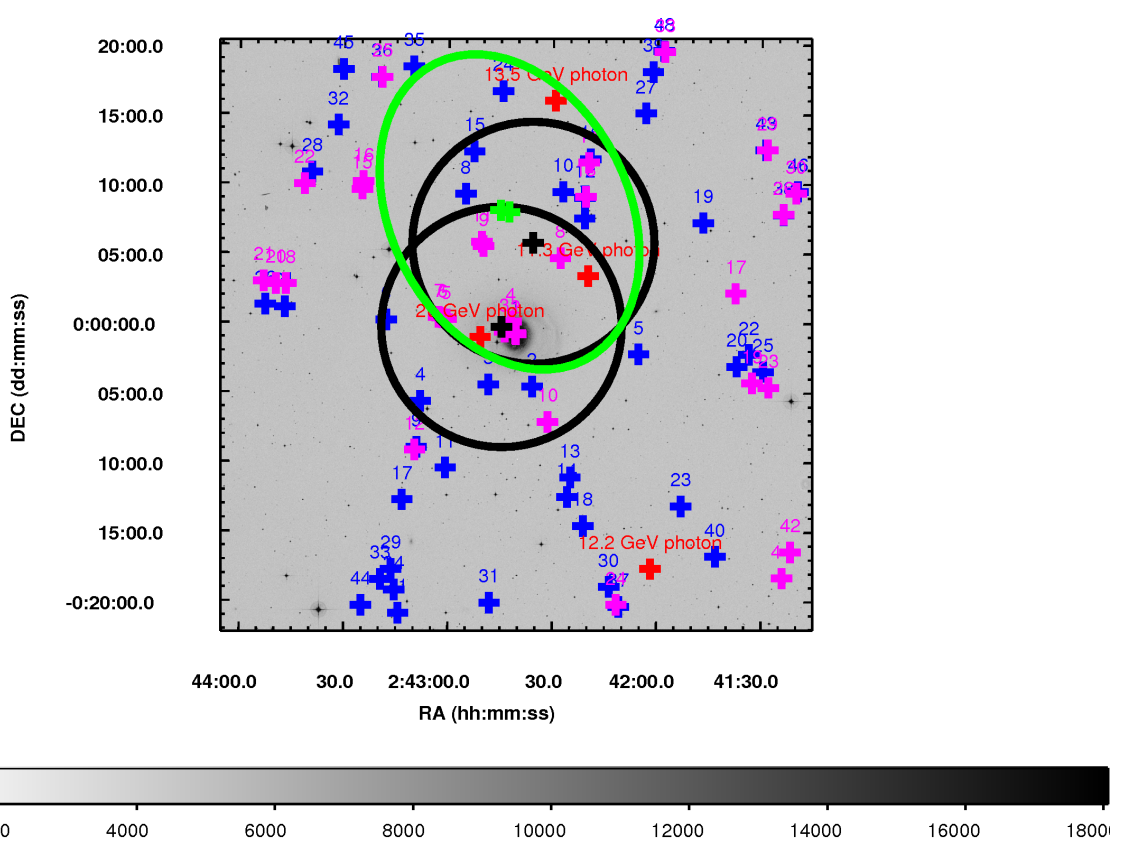

Figure 1: Optical skymap of NGC 1068. FIRST (magenta, [Becker et al.(1997)]) and NVSS (blu, NRAO VLA Sky Survey [Condon et al.(1998]]) sources overlaid. The green ellipse represents the error circle on the localization of 1FGL J0242.7+0007 in the 11 months Fermi catalog. The green cross points represents the centroid of the Fermi published position and the centroid of the cross-checked position from our analysis of the 11 months data set. The black circles represent the $95 \%$ contour on the best fit localization from $t$ he analysis presented in this study (higher declination: all energy, lower declination: $\mathrm{E}>10 \mathrm{GeV}$ ), with their centroid as a black cross. Red crosses represent the reconstructed arrival direction of the highest energy LAT photons. 


\subsection{Spectrum and Lightcurve Details}

The spectral analysis has been performed through an unbinned likelihood fit (gtlike tool in the public software). A likelihood fit on the entire data range was made with a power law functional form $d N / d E=N_{0}\left(E / E_{\mathrm{p}}\right)^{-\Gamma}$, where $N_{0}$ is the normalization at the pivot energy Ep and $\Gamma$ is the spectral index. The background has been modelled as being composed from the sources in the first year Fermi Catalog (in a $25^{\circ}$ region centered on the nominal position of NGC 1068) and from two diffuse backgrounds. The diffuse backgrounds (both galactic and extragalactic) have been modelled from the ones supplied along with the public software. Even though the source is not in the vicinity of the galactic plane $(1, \mathrm{~b}=172.1045,-51.9336)$, the diffuse emission is always dominant in counts. Indeed almost $10^{5}$ photons come from extragalactic and galactic diffuse background, but this is to be expected [Abdo et al.(2010]]. The best fit power-law for the photon spectrum is $F(>200 \mathrm{MeV})=\left(4.8_{-1.3}^{+1.7}\right) \times 10^{-9} \mathrm{~cm}^{-2} \mathrm{~s}^{-1}$, with a spectral index of $\Gamma=2.3 \pm 0.16$.

The systematic uncertainties on the effective area, evaluated by comparing the efficiencies of analysis cuts for data and simulation of observations of Vela, are energy dependent: $10 \%$ at 100 $\mathrm{MeV}$, decreasing to $5 \%$ at $560 \mathrm{MeV}$, and increasing to $20 \%$ at $10 \mathrm{GeV}$ and above [Abdo et al.(2009]]. It is therefore consistent with the spectrum given in the $1 \mathrm{FGL}$ catalog. The $\chi^{2}$ corresponding to the spectrum given here is $\chi^{2}=7.2\left(\chi^{2} / \mathrm{dof}=7.2 / 4\right)$. This is obtained fixing the normalization and the spectral index obtained from the pipeline likelihood maximization and fitting those values to the binned spectral points. There is no evidence for deviation from a simple power law behavior. The gamma-ray flux is shown in Fig. \along with the multiwavelength data from the core of NGC 1068 .

In order to search from variability in the signal, a counts lightcurve was constructed. From the cumulative lightcurve there is no indication for a preferred timescale, therefore the counts inside the psf extension have been binned in 60 days bins. There is no strong indication for variability as expected. However a decrease in the flux cannot be excluded.

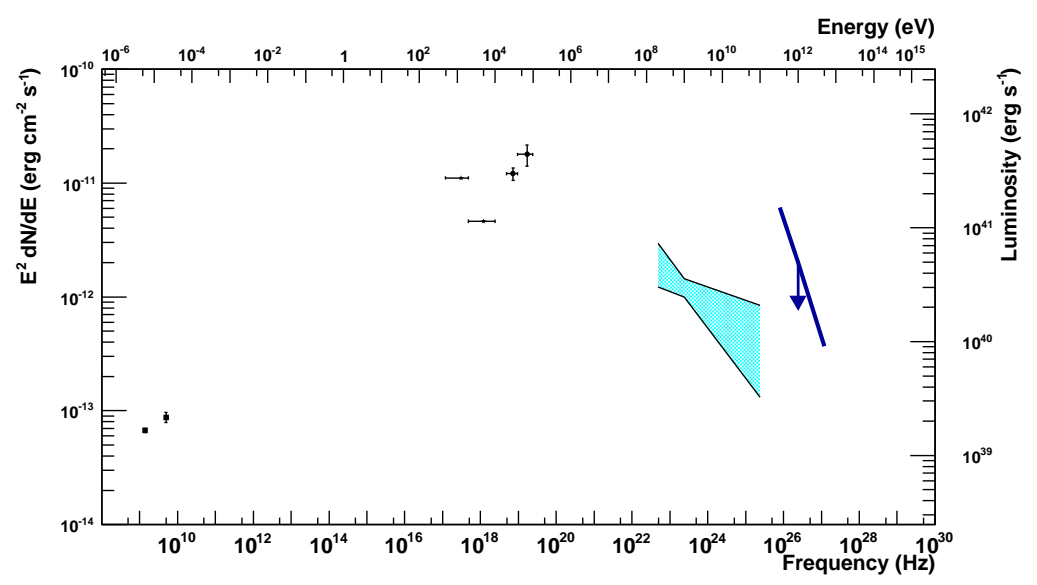

Figure 2: The SED of NGC 1068. The black squares are VLA data [Wilson \& Ulvestad(1987]], black stars are XMM data [Cappi et al.(2006)] and black dots are Integral data [Bird et al.(2007)]. The cyan butterfly are the Fermi data presented in this paper. The blue dots are the H.E.S.S. spectrum $99.9 \%$ upper limit [Aharonian et al. (2008]. 


\section{Identification of NGC 1068}

In the $95 \%$ confidence contour of the best fit position obtained in this study from gammarays, other radio sources are present. In Fig. $\mathbf{W}^{1}$, are shown the objects belonging to the FIRST [Becker et al.(1997)] and NVSS (NRAO VLA Sky Survey [Condon et al.(1998]]) catalogs. Among those, the ones that are believed to be aliases or components of NGC 1068 itself were removed, leading to a sample of possible counterparts listed in Table $\mathbb{W}$. Among the possible counterparts, only NGC 1068 is catalogued in all investigated energy regimes (radio, X-ray with XMM catalog data and optical from SDSS). Moreover, the other possible counterparts show a much lower flux in the radio domain, with a ratio between 2 and 3 orders of magnitude to NGC 1068 flux.

\begin{tabular}{|c|c|c|c|c|c|}
\hline RA & DEC & FIRST & NVSS & XMM & SDSS \\
\hline 2h42m40.82400s & $-0 \mathrm{~d} 00 \mathrm{~m} 48.3998 \mathrm{~s}$ & 1,2 & 1 & $\checkmark$ & $\checkmark$ \\
\hline 2h42m27.91200s & $+0 \mathrm{~d} 04 \mathrm{~m} 40.5098$ & 8 & - & $\checkmark$ & $\checkmark$ \\
\hline 2h43m08.01600s & $-0 \mathrm{~d} 05 \mathrm{~m} 37.9000 \mathrm{~s}$ & - & 4 & $\checkmark$ & - \\
\hline 2h42m20.68800s & $+0 \mathrm{~d} 09 \mathrm{~m} 05.2812 \mathrm{~s}$ & 13 & 12 & - & - \\
\hline 2h42m20.97600s & $+0 \mathrm{~d} 07 \mathrm{~m} 31.0008 \mathrm{~s}$ & - & 7 & - & - \\
\hline 2h42m27.19200s & $+0 \mathrm{~d} 09 \mathrm{~m} 25.8012 \mathrm{~s}$ & - & 10 & - & - \\
\hline 2h42m19.80000s & $+0 \mathrm{~d} 11 \mathrm{~m} 31.7112 \mathrm{~s}$ & 14 & 16 & $\checkmark$ & - \\
\hline
\end{tabular}

Table 1: Possible association with the gamma-ray excess. The numbers refer to Fig. $\mathbb{W}$. All the objects have XMM or optical objects close-by. Here were considered only objects whose distance is less than 30 ”.

\section{Conclusions}

Fermi LAT data reveal a gamma-ray source coincident with the position of the Seyfert 2 galaxy NGC 1068. This excess is already part of the First year Fermi catalog, but the counterpart is not yet identified. Here, an analysis of an extended set of LAT data was analyzed with results consistent with what derived in the catalog. Thanks to the extended exposure, both spectrum determination and positional analysis were refined with respect to the catalog.

There is no indication of relativistic motion in the system that could lead to a relativistic boosting of the emission. However, the gamma-ray luminosity inferred from the LAT data and a distance of maximum $\mathrm{D}=14.4 \mathrm{Mpc}$ [एully(1988)] can easily be sustained by the kinetic jet power of $P_{\text {jet }}>3 \times 10^{43} \mathrm{erg} \mathrm{s}^{-1}$.

While other possible counterparts emerge from a search in the most complete radio catalogs, no firm evidence of association exists.

\footnotetext{
${ }^{1}$ Based on photographic data obtained using The UK Schmidt Telescope. The UK Schmidt Telescope was operated by the Royal Observatory Edinburgh, with funding from the UK Science and Engineering Research Council, until 1988 June, and thereafter by the Anglo-Australian Observatory. Original plate material is copyright (c) of the Royal Observatory Edinburgh and the Anglo-Australian Observatory. The plates were processed into the present compressed digital form with their permission. The Digitized Sky Survey was produced at the Space Telescope Science Institute under US Government grant NAG W-2166.
} 


\section{References}

[Abdo et al.(2009)] Abdo, A. A. et al. 2009, ApJS, 170, 175B

[Abdo et al.(2010)] Abdo, A. A. et al. 2010 PhRevL, 104, 101101

[Aharonian et al.(2008)] Aharonian, F. et al. (H.E.S.S. Collaboration) 2008, A\&A, 478, 387

[Becker et al.(1997)] Becker, R.H. et al. 1997, ApJ, 475, 479W

[Bird et al.(2007)] Bird, A. J. et al. 2007, ApJS, 170, 175B

[Cappi et al.(2006)] Cappi, M. et al 2006, A\&A, 446, 459C

[Condon et al.(1998)] Condon J.J. et al. 1998 AJ, 115, 1693C

[Fath(1909)] Fath, E. A. 1909, LicOB, 5, 71F

[Lenain et al.(2010)] Lenain et al. 2010, A\&A, 524A, 72L

[Seyfert(1943)] Seyfert, C. K. 1943, ApJ, 97, 28S

[Tully(1988)] Tully, R. B. 1988 NBGC

[Wilson \& Ulvestad(1987)] Wilson, A. S. \& Ulvestad, J. S. 1987, ApJ, 319, 105

[Wilson \& Ulvestad(1987)] Wilson, A. S. \& Ulvestad, J. S. 1983 ApJ, 275, 8W 